\title{
Rise of Lower GI Complaints in Young Population: 5 Year Detailed Analysis of Colonoscopic Findings Using ASGE Guidelines in Public Center Hospital of Pakistan
}

\author{
RAMISH RIAZ1 1 TANVEER AHMED ${ }^{2}$, SHAH RUKH ABBAS ${ }^{3}$, IMRAN YOUSUF 4 \\ ${ }^{1}$ Visiting Lecturer, Radiology Department, Pakistan Institute of Medical Sciences, Islamabad\& Rawalpindi Medical University, Rawalpindi \\ ${ }^{2}$ Post Graduate Resident, MD Gastroenterology, Department of Pakistan Institute of Medical Sciences, Islamabad \\ ${ }^{3}$ Assistant Professor, Atta ur Rahman School of Applied Biosciences, National University of Science \& Technology, Islamabad \\ ${ }^{4}$ Registrar, Gastroenterology Department, Pakistan Institute of Medical Sciences, Islamabad \\ Corresponding Author: Dr. Tanveer Ahmed \\ Email Address: ahmedtanveer17@yahoo.com, Cell No.+923327201463
}

\begin{abstract}
Introduction: Colonoscopy gives detail account of lower gastro intestinal tract pathologies. As the procedure is invasive, operator dependent and associated with few complications, clear indication has to be established.Current study was conducted to evaluate detailed account of clinicopathological findings on colonoscopy that will give idea about the prevalent lower gastrointestinal tract disorders, common complaints, correlation between findings and symptoms, age and gender wise distribution of these pathologies among our population. The study will serve as baseline to construct nationwide guidelines for the procedure.

Materials \& Methods: This retrospective study was carried out at Gastroenterology Department, Pakistan Institute of Medical Sciences from January 2014 to June 2019. All the patients fulfilling the ASGE criterion or having complaints of lower Gl tract for more than 2 months underwent colonoscopy. Patients with inadequate preparation or other undergoing therapeutic procedures were excluded from the study. Total 850 patients fulfilling the criterion were included in the study. Data was recorded and analyzed by SPSSv20.

Results: Mean age of the patients was $43.02 \pm 18.22$. Majority (63.9\%) of the patients were males. Majority $(n=543,63.9 \%)$ of the patients had some finding on colonoscopy. Most common finding on colonoscopy was hemorrhoid followed by ulceration, mass, inflammation and polyps.Most common complaint was per rectum bleed followed by diarrhea, suspected growth on other modalities andsuspected inflammatory bowel disease.Total 540 patients i.e. $63.52 \%$ were below age of 50 years.. Trend of pathologies showed ulceration to be most common finding in patients below 50 years followed by hemorrhoids and mass.

Conclusion: The results of our finding shows rise of lower Gl pathologies in young male population of Pakistan with ulcerative colitis, hemorrhoids and colorectal cancer being top three diseases. This is an alarming situation and we need to take urgent big steps to control it.

Keywords: Colonoscopy, Hemorrhoids, Colorectal Cancer, Per Rectal Bleed, Ulceration
\end{abstract}

\section{INTRODUCTION}

Colonoscopy is nowadays widely used for diagnosis of colon disorders. When performed accurately, it is a safe and well tolerable procedure giving detailed evaluation of entire colonic mucosa and allowing few therapeutic procedures.(1) Procedure allows the diagnosis of inflammatory changes detecting benign erythema to ulceration, tumors, luminal narrowing and dilatations, strictures, hemorrhoids and motility problems. Therapeutic procedure that could be performed via colonoscopy includes polypectomy, stent placement, dilatation of stricture, and removal of foreign body, sclerotherapy, banding and taking sample for biopsy. Pictures can be taken in real time and video can be recorded for future use.(2)

With rise of CRC worldwide, use of colonoscopy has further increased.Screening colonoscopy usually begins at 50 years of age.(3) However in Pakistan young onset CRC is more common with studies showing mean age to be 40 to 45 years. Most of the cancers present with painless per rectum bleed, therefore gastroenterologist in our country perform colonoscopy in all such cases. (4)As the country lacks any nationwide Cancer Registryor Gastroenterology Society to recommend guidelines for appropriate use of colonoscopy, decision of colonoscopy is usually made on
American Society for Gastrointestinal Endoscopy (ASGE) or European Panel on Appropriateness of Gastrointestinal Endoscopy (EPAGE) II recommendations. However there is no strict following, some patients not fulfilling the criterion but having prolonged symptoms are also considered for colonoscopy.

According to provincial level Punjab Cancer Registry Report, CRC is the third common cancer in Pakistani Population. (5)Despite of this high rate, country still lack any screening program. There is lack of awareness among the people regarding its symptoms, need of colonoscopy, also patients are usually hesitant to undergo the procedure especially females due to feeling embarrassment associated with the procedure. Also the procedure is expensive which makes it out of the reach of majority of the population living in rural areas.(3)

According to ASGE guidelines, indication for colonoscopy includes any abnormality seen on other modalities needing further evaluation, unexplained GI bleed, screening purposes, significant diarrhea of unexplained origin, suspected chronic inflammatory bowel disease, treatment of hemorrhoids, polypectomy and taking sample for biopsy. Inappropriate referrals include chronic abdominal pain, acute diarrhea, constipation, fulminant 
colitis, and routine followup of inflammatory Bowel Disease.(2)

Adequate preparation of bowel is one of the quality indicators of colonoscopy. It affects the completeness of procedure, need of reevaluation and procedure duration. Patient must be guided appropriately regarding bowel preparation.(1)In some cases flexible sigmoidoscopy could be done which includes examination of rectum, sigmoid colon and variable portion of proximal colon.(2)

Colonoscopy is generally a well tolerated procedure, however $33 \%$ of the patients report minor complaint mainly cramps in abdomen and bloating. Major risk associated with colonoscopy is risk of perforation, however its occurrence is low, $0.3 \%$ in therapeutic procedures and less than $0.01 \%$ in diagnostic procedures. Other complications which can be encountered include hemorrhage, diverticulitis, gas explosion and cardiopulmonary complications due to sedation. Overall rate of serious complication is 2.8 per 1000 procedures.(6)

Current study was conducted to evaluate detailed account of clinicopathological findings on colonoscopy giving idea about the prevalent lower Gl disorders, common complaints, correlation between findings and symptoms, age and gender wise distribution among our population. The study will serve as baseline to construct nationwide guidelines for the procedure.

\section{MATERIAL AND METHODS}

This retrospective study was carried out at Gastroenterology Department, Pakistan Institute of Medical Sciences from January 2014 to June 2019. All the patients fulfilling the ASGE criterion were considered for colonoscopy. Few patients not fulfilling the criterion but having complaints of lower Gl tract for more than 2 months also underwent colonoscopy.All patients in which colonoscopy could not be completed due to inadequate preparation or other reason were excluded from the study. Patient undergoing therapeutic procedure were excluded from the study. Total 850 patients fulfilling the criterion were included in the study.

For bowel preparation, standard guidelines by ASGE were adapted. Colonoscopy was performed by consultant gastroenterologist. Reports were recorded. Firstly data was arranged and values label were given in SPSS v 20. ODDS Ratio and Relative Risk Tests were applied to check associations. Dispersion in quantitative data was analyzed by standard deviation. Statistical significance was checked by $\mathrm{P}<0.05$ values.

\section{RESULTS}

Mean age of the patients was $43.02 \pm 18.22$. Majority $(n=520,63.9 \%)$ of the patients were males. Majority $(n=543,63.9 \%)$ of the patients had some finding on colonoscopy. 307 patients $(36.1 \%)$ had normal colonoscopy. Most common finding on colonoscopy was hemorrhoids $(n=150,17.6 \%)$ followed by ulceration $(n=118$, $12.8 \%)$, mass $(n=109,13.8 \%)$, inflammation $(n=70,8.2 \%)$ and polyps $(n=50,5.9 \%)$. Other findings include mild erythema $(n=15,1.8 \%)$, diverticuli $(n=9,1.1 \%)$, strictures $(n=8,0.9 \%)$, fistula $(n=3,0.4 \%)$, volvulus $(n=3,0.4 \%)$, Hirshprung $(n=2,0.2 \%)$, fissure $(n=2,0.2 \%)$, dilatation $(n=2,0.2 \%)$, worms $(n=1,0.1 \%)$ and abscess $(n=1,0.1 \%)$.
Most common complaint was per rectum bleed $(n=392$, $46.1 \%)$ followed by diarrhea $(n=127,14.6 \%)$, suspected growth $(n=118,13.6)$ on other modalities, suspected IBD $(n=50,5.9 \%)$, metastatic disease, constipation, post operative surveillance, pain, altered bowel habits, weight loss, pain, suspected TB, anemia, tenesmus and acute abdomen.

Most common site for mass was rectum (38.5\%) followed by rectosigmoid junction, ascending colon, cecum, sigmoid colon and transverse colon.Most common site for ulceration was also rectum (40\%) followed by rectosigmoid junction, ileocecal junction and ileum.Majority (68.8\%) of the hemorrhoids were internal.

Total 540 patients i.e. $63.52 \%$ were below age of 50 years. Among young age group, $62.8 \%$ of the studies had pathology while in older group $65.18 \%$ were abnormal. No significant difference $(O R=1.14, R R=1.04 \quad P=0.3)$ was found between age and lower Gl tract pathology. Trend of pathologies showed ulceration to be most common finding in patients below 50 years followed byhemorrhoids, mass and inflammation while in patients above 50 years most common finding was hemorrhoid followed by mass ulceration and polyps (Error! Reference source not found.). Trend of pathologies on the basis of pathologies showed hemorrhoids to be the most common finding followed by mass and ulceration, while among females, ulceration was the commonest finding followed by hemorrhoid and mass (Table-1)

Table No 1: Gender-wise distribution of pathologies

\begin{tabular}{|l|l|l|l|}
\hline \multirow{2}{*}{ FINDINGS } & Gender & \multirow{2}{*}{ Total } \\
\cline { 2 - 3 } & Male & Female & \\
\hline Normal & 199 & 108 & 307 \\
\hline Mass & 67 & 42 & 109 \\
\hline Polyp & 37 & 13 & 50 \\
\hline Abscess & 0 & 1 & 1 \\
\hline Diverticuli & 5 & 4 & 9 \\
\hline Ulceration & 61 & 57 & 118 \\
\hline Erythema & 7 & 8 & 15 \\
\hline Hemorrhoids & 96 & 54 & 150 \\
\hline Inflammation & 36 & 34 & 70 \\
\hline Fissure & 1 & 1 & 2 \\
\hline Volvulus & 2 & 1 & 3 \\
\hline Hirshprung & 0 & 2 & 2 \\
\hline Fistula & 2 & 1 & 3 \\
\hline Dilation & 2 & 0 & 2 \\
\hline Worms & 4 & 4 & 8 \\
\hline worms & 1 & 0 & 1 \\
\hline Total & 520 & 330 & 850 \\
\hline
\end{tabular}

Figure 1: Symptoms wise distribution

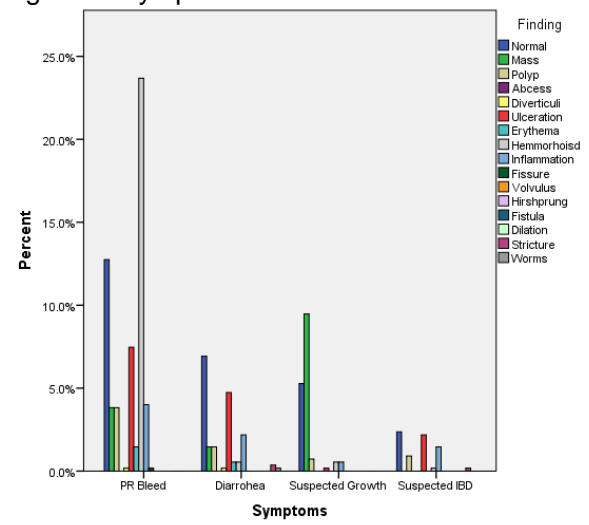


According to ASGE guidelines, 789 cases i.e. $92.9 \%$ of the referrals were appropriate, 61 cases i.e. $7.2 \%$ were in appropriate. Most common reason for inappropriate referral was constipation followed by pain and tenesmus. Among appropriate referrals 516 i.e. $60.7 \%$ had pathology on colonoscopy, while among inappropriate referrals, 27 cases i.e. $44.2 \%$ had abnormal finding. Significant association ( $O R=2.38, R R=1.47, P=0.001)$ was found between appropriate referral and finding disease. Among four major indications for colonoscopy, majority of the PR bleeds patients had hemorrhoids, patients with suspected mass on other findings had mass on colonoscopy, patients with diarrhea and suspected IBD were normal.

We also analyzed the data to see the trend of pathologies with time. Highest number $(n=229)$ of colonoscopies were done in 2015 followed by 2016 ( $n$ $=189), 2018(n=173), 2017(n=130)$ and $2014 \quad(n=129)$ (Table-2). In 2014, 2015, 2016 and 2017 most common finding was hemorrhoids, but in 2018 most common finding was ulceration followed by mass and hemorrhoids (Table 2).

Table No 2: Year wise-distribution

\begin{tabular}{|c|c|c|c|c|c|c|}
\hline \multirow[t]{2}{*}{ FINDINGS } & \multicolumn{5}{|l|}{ Year } & \multirow{2}{*}{ Total } \\
\hline & 2018 & 2017 & 2016 & 2015 & 2014 & \\
\hline Normal & 72 & 37 & 54 & 93 & 51 & 307 \\
\hline Mass & 21 & 20 & 32 & 26 & 10 & 109 \\
\hline Polyp & 13 & 12 & 17 & 6 & 2 & 50 \\
\hline Abscess & 1 & 0 & 0 & 0 & 0 & 1 \\
\hline Diverticuli & 3 & 2 & 2 & 1 & 1 & 9 \\
\hline Ulceration & 25 & 20 & 31 & 26 & 16 & 118 \\
\hline Erythema & 3 & 6 & 3 & 3 & 0 & 15 \\
\hline Hemorrhoids & 19 & 23 & 33 & 44 & 31 & 150 \\
\hline inflammation & 13 & 9 & 10 & 26 & 12 & 70 \\
\hline fissure & 2 & 0 & 0 & 0 & 0 & 2 \\
\hline volvulus & 0 & 1 & 1 & 1 & 0 & 3 \\
\hline Hirshprung & 0 & 0 & 2 & 0 & 0 & 2 \\
\hline Fistula & 0 & 0 & 2 & 0 & 1 & 3 \\
\hline Dilation & 1 & 0 & 1 & 0 & 0 & 2 \\
\hline stricture & 0 & 0 & 1 & 2 & 5 & 8 \\
\hline worms & 0 & 0 & 0 & 1 & 0 & 1 \\
\hline Total & 173 & 130 & 189 & 229 & 129 & 850 \\
\hline
\end{tabular}

\section{DISCUSSION}

Colonoscopy is now a routine procedure for evaluation of lower Gl tract pathologies. As the procedure is invasive, operator dependent and associated with few complications, clear indication must have to be established. In most of the world guidelines by American Society for Gastrointestinal Endoscopy (ASGE) or European Panel on Appropriateness of Gastrointestinal Endoscopy (EPAGE) II are used. As Pakistan lacks any nationwide gastroenterology society, ASGE guidelines are usually adopted. (2)

However dynamic of Lower Gl tract pathologies in Pakistan is very different. In western world, mean age of patientsundergoing colonoscopy due to lower $\mathrm{Gl}$ complaints is around 55 years with most common finding of polyps however in our study mean age of population was 43 years. Comparative study between American and Chinese population showed mean age of Chinese population to be 45 years. (7)The similar change in trend has also been noted in age of CRC in Asian countries versus western world where it is considered to be a disease of old age with onset from 50 to 70 years. While in Pakistan young onset $\mathrm{CRC}$ is at rise with mean age of CRC to be 45 . Also sporadic non polyposis CRC is common among our population and usually diagnosed when disease is already advanced. While in west familial adenomatous polyposis CRC is common, this could be one of the reasons behind polyps being common finding in western population. Also it is associated with old age colorectal cancer.In Pakistan, left sided CRC is more common with rectum being most common site and $\mathrm{PR}$ bleed as most common feature of presentation.(4) Our results also showed similar findings.

Lower Gl bleed is the most common indication for colonoscopy. It is usually due to benign reasons due to anorectal diseases. Most common cause is hemorrhoids. (8)Each year 4 million cases of hemorrhoids are diagnosed in America. It is the third most common outpatient Gl diagnosis. Resultsfrom American study showed, females to be more affected by hemorrhoids than males. Among GI disorders, it is the most common cause of visit to the hospital even more common than inflammatory bowel disease, diverticular disease or colon cancer. (9) Another study conducted in Singapore also showed hemorrhoids to be the major finding followed by Polyps with male preponderance.(10)Our results also showed hemorrhoids to be most common finding with male preponderance. Our results were also in concord with the study by Irfan et al who find internal hemorrhoid to be most common finding on sigmoidoscopy. (11)

Worldwide, inflammatory bowel diseases including ulcerative colitis and crohn disease are the significant contributors to disease burden. In UK, annual incidence of IBD is around 13000.(12) Previously considered as disease of western countries, prevalence of IBD has increased remarkably in Asia, with East Asian countries being most affected. The reason might be westernization of our diet. In our study, ulceration was the second common finding over all and commonest finding among patients aged below 50 . This change in trend has also been noted in Pakistan. Study conducted in same city of Pakistan in 2013 showed 
ulcerative colitis to be $4^{\text {th }}$ common finding with $8.4 \%$ prevalence. However result of our study in year 2018 showed ulceration to be most common finding with frequency of $14.5 \%$.(13)

Results of our finding shows rise of lower GI pathologies in young male population of Pakistan with ulcerative colitis, hemorrhoids and colorectal cancer being top three diseases. There is an urgent need of development of Gastroenterology Society which could look into this situation and develop guideline according to country population, as western guidelines are mostly developed for their population where most of the $\mathrm{Gl}$ problems are associated with old age. Also our study was a single centre study. There is an urgent need to conduct such study on national scale, as we did not have any baseline record data. Also frequency of $C R C$ in our study population was $12.8 \%$ which is quite high and need urgent attention. Unfortunately not a single patient in a study underwent colonoscopy for screening purpose. There is a need to increase awareness among population regarding $\mathrm{CRC}$ and need for screening. This is an alarming situation and we need to take urgent big steps to control it.

\section{CONCLUSION}

Majority $(63.9 \%)$ of the patients undergoing colonoscopy had abnormal finding. The results of our finding shows rise of lower Gl pathologies in young male population of Pakistan with ulcerative colitis, hemorrhoids and colorectal cancer being top three diseases. This demands an urgent need urgent need for development of Gastroenterology Society which could look into this situation and develop guideline according to country populationand take appropriate steps to solve theexistentalarming situation.

\section{REFERENCES}

1. Rex, D. K., Schoenfeld, P. S., Cohen, J., Pike, I. M., Adler, D. G., Fennerty, M. B., et al. Quality indicators for colonoscopy. Gastrointestinal Endoscopy. 2015; 81 (1): 3153.

2. Early, D. S., Ben-Menachem, T., Decker, G. A., Evans, J. A., Fanelli, R. D., Fisher, D. A., et al. Appropriate use of Gl endoscopy. Gastrointestinal Endoscopy. 2012; 75 (6): 11271131.

3. Hasan, F., Shah, S. M., Munaf, M., Khan, M. R., Marsia, S., Haaris, S. M., et al. Barriers to Colorectal Cancer Screening in Pakistan. Cureus. 2017; 9 (7): e1477.

4. Riaz, R., Masood, N., \& Benish, A. Red flag symptoms: detailed account of clinicopathological features in youngonset colorectal cancer. Intestinal Research. 2017; 15 (2): 203-207.

5. Punjab Cancer Registry.Punjab cancer registry report-4. Lahore : Punjab Cancer Registry., 2016.

6. Fisher, D. A., Maple, J. T., Ben-Menachem, T., Cash, B. D., Decker, G. A., Early, D. S., et al. Complications of colonoscopy. Gastrointestinal Endoscopy. 2011; 74 (4): 74552.

7. Wang, H., Cai, Q., Zhu, H. T., Hua Lv, N., \& Zhu, X. A comparative analysis of colonoscopy findings in a Chinese and American tertiary hospital. Turk J Gastroenterol. 2015; 26: 263-9.

8. Khodadoostan, M., Shavakh, A., Padid, R., Shavakhi, A., \& Ahmadian, M. Full colonoscopy in patients under 50 years old with lower gastrointestinal bleeding. J Res Med Sci. 2018; 23: 45-53.

9. Sandler, R. S., \& Peery, A. F. Rethinking What We Know About Hemorrhoids. Clinical Gastroenterology and Hepatology. 2019; 17: 8-15.

10. Koh, F. H., Seah, A., Chan, D., Ng, J., \& Tan, K.-K. Is Colonoscopy Indicated in Young Patients with Hematochezia? Gastrointest Tumors. 2017; 4: 90-95.

11. Irfan, M., Saleem, A., Batool, S., Aftab, G. M., Naqvi, A., \& Mohsin, A. Internal Hemorrhoids and Sigmoidoscopy; A Retrospective Analysis of Grades and Other Anorectal Disorders. P J M H S. 2018; 12 (2):561-63.

12. Stapley, S. A., Rubin, G. P., Alsina, D., Shephard, E. A., Rutter, M. D., \& Hamilton, W. T. Clinical features of bowel disease in patients aged $<50$ years in primary care:a large case-control study. British Journal of General Practice.2018; 67 (658): e336-e344.

13. Rehman, K. U., Qureshi, M. O., Khokar, N., Shafqat, F., \& Salih, M. Quality of Colonoscopy and Spectrum of Lower Gastrointestinal Disease as Determined by Colonoscopy. Journal of the College of Physicians and Surgeons Pakistan. 2015; 25 (7):478-481. 